\title{
Remote and Autonomous Controlled Robotic Car based on Arduino with Real Time Obstacle Detection and Avoidance
}

\author{
Esra Yılmaz, Sibel T. Özyer ${ }^{*}$ \\ Department of Computer Engineering, Çankaya University, Turkey
}

Copyright@2019 by authors, all rights reserved. Authors agree that this article remains permanently open access under the terms of the Creative Commons Attribution License 4.0 International License

\begin{abstract}
In robotic car, real time obstacle detection and obstacle avoidance are significant issues. In this study, design and implementation of a robotic car have been presented with regards to hardware, software and communication environments with real time obstacle detection and obstacle avoidance. Arduino platform, android application and Bluetooth technology have been used to implementation of the system. In this paper, robotic car design and application with using sensor programming on a platform has been presented. This robotic device has been developed with the interaction of Android-based device. Arduino Uno has been used as the robot's brain. The robot has many hardware components such as Bluetooth module, PIR sensor, ultrasonic sensor, and buzzers. It also consists of the software component that uses a mobile application. The desired direction or mode by mobile application can be selected by the user of the robotic car to control the movement of the car. The user can control the robot movements from his/her own intelligent device or take the robot in automatic mode and let the car drive its own way. Thus, the robot can flee from the obstacle and also detect live objects. The purpose of this article is to alert the civilian and military personnel to potential terrorist attacks especially in military areas with live detectable sensors.
\end{abstract}

Keywords Android-based Devices, Robotic Car based on Arduino, Obstacle Detection, Obstacle Avoidance

\section{Introduction}

In the last decade, with the development of technology, sensors used with electronic devices have been used in many areas to facilitate life. Sensors are devices that convert energy forms into electrical energy. The sensors serve as a bridge connecting the environment and various electronic devices. The environment can be any physical environment such as military areas, airports, factories, hospitals, shopping malls, and electronic devices can be smartphones, robots, tablets, smart clocks. These devices have a wide range of applications to control, protect, image and identification in the industrial process. Today, there are hundreds of types of sensors produced by the development of technology such as heat, pressure, obstacle recognizer, human detecting. Sensors were used for lighting purposes in the past, but now they are used to make life easier. Thanks to technology in the field of electronics, incredibly fast developments are experienced. In this respect, it is possible to develop a new invention or a new application in every day and make life easier.

Today, robot systems are developed with the use of artificial intelligence algorithms. The robotics field is one of them. The most important part of the robot is the perception. Perceive of the environment will be important for a robot design. For instance, it is very important to identify explosives by a robot to detect a terrorist in the military field by using sensors. A robot has to perceive some variables (like heat changes) around it, interpret it, and then decide to act accordingly.

In this article, remote and autonomous controlled robotic car has been presented in terms of obstacle detection and avoidance by using sensors. The connection between the robot and the Android device has been established via Bluetooth technology. The incoming data will be processed by Arduino Uno and according to the input value of the user, robot action can be performed. There are two main modes that control the robotic car by Android application (mobile phone). These are user control mode and automatic mode. A menu with buttons has been seen on the screen to select the actions. These buttons will be used to move the robotic car forward, backward, right and left, stopping the car and switching to automatic mode. By selecting automatic mode, the user leaves the robot control and the robot finds its way without hitting the obstacles. The robot 
detects living beings that are encountered and gives warning. The robot finds its way without hitting spot and when it comes to the obstacle it perceives and stops. Concurrently, it makes live detection with the temperature sensor and gives warning with red led on it.

In this study, we carry out a novel vehicle design with real time obstacle detection and avoidance. To best of our knowledge, there is no previous study that investigates real time obstacle detection and avoidance by using Arduino Uno and Android Platform.

This work is as follows. Section-2 deals with related works in this regard. Section-3 describes the working principles of the robotic car and system architecture. Section-4 shows the specifications of the robotic car and the materials used. Section-5 is design and implementation of the robotic car. Section- 6 gives conclusion of the paper.

\section{Literature Review}

In this section, similar studies in the past have been explored and some of the methods and working principles used are summarized.

S. S. Pujari et al. [1] designed a Robot for the working families that could monitor children remotely and communicate with the camera. Raspberry Pi 3, camera module, Wifi and Bluetooth technology used by the robot. For Raspberry Pi, the heart was defined as the Robot and used the Phyton language to code it.

M. R. Mishi et al. [2] designed a robotic car. Arduino Uno and Raspberry Pi were used together to control robot in this project. GPS was also used to trace the car and the distances between the obstacle and the path are measured. The data in the cloud was used without having to be online. Thus, the multi-motion system was controlled.

D. Chakraborty et al. [3] designed and developed a robotic car using sensors and Bluetooth technology. They had established communication between smart device and the robot. Thanks to the phone camera, they had observed the living beings. The obstacles in the opposite direction were prevented from colliding with the ultrasonic ranging sensor. Images recorded with the camera were recorded in the database and analyzed.

S. J. Lee et al. [4] designed an autonomous robotic car used Arduino Uno R3 for robot's brain. Also, Bluetooth module and the ultrasonic sensor had been used in this paper. The robot scanning the placed QR codes could move along the road in autonomous form thanks to the QR codes. It also provided voice communication with the Android device in the Text-to-speech feature. It also moved with the help of an ultrasonic sensor without hitting the objects around it. In this view, range information was collected. In order for the motion of the robot to be smooth, the deviation was minimized by the PID algorithm.

E. Amareswar et al. [5] designed a robot used for the military area. Thanks to the metal detector, the robot played an important role in the detection of explosives, and the surroundings could be viewed thanks to the camera of the used Android device. This robot system consisted of Android device, Bluetooth module, a microcontroller (Arduino Uno), DC motors, motor driver, wireless camera and metal detector.

Premkumar et al. [6] designed robotic arm controlled using Raspberry Pi. The main purpose of this robot was to add the human arm feature to the robot arm. Raspberry Pi was the code written in the Python language that provided arm movement. With the Android application, the user moved the robot arm in the desired direction. Robotic arm control was provided in this way. The Android app was written in Java. Thus, the communication between the Android application and Raspberry Pi was provided by the Wi-Fi connection. This communication moved the robot arm to the right and left.

In this study, real time obstacle detection and avoidance with remote and autonomous controlled robotic car based on Arduino has been carried out by using Android application.

\section{System Architecture}

The system structure of the developed robotic car using various sensors is shown in Figure 1.

In this paper, the robotic car consists of two modes: The user control and Automatic mode. The robot and Android device communicate with each other using Bluetooth technology. 


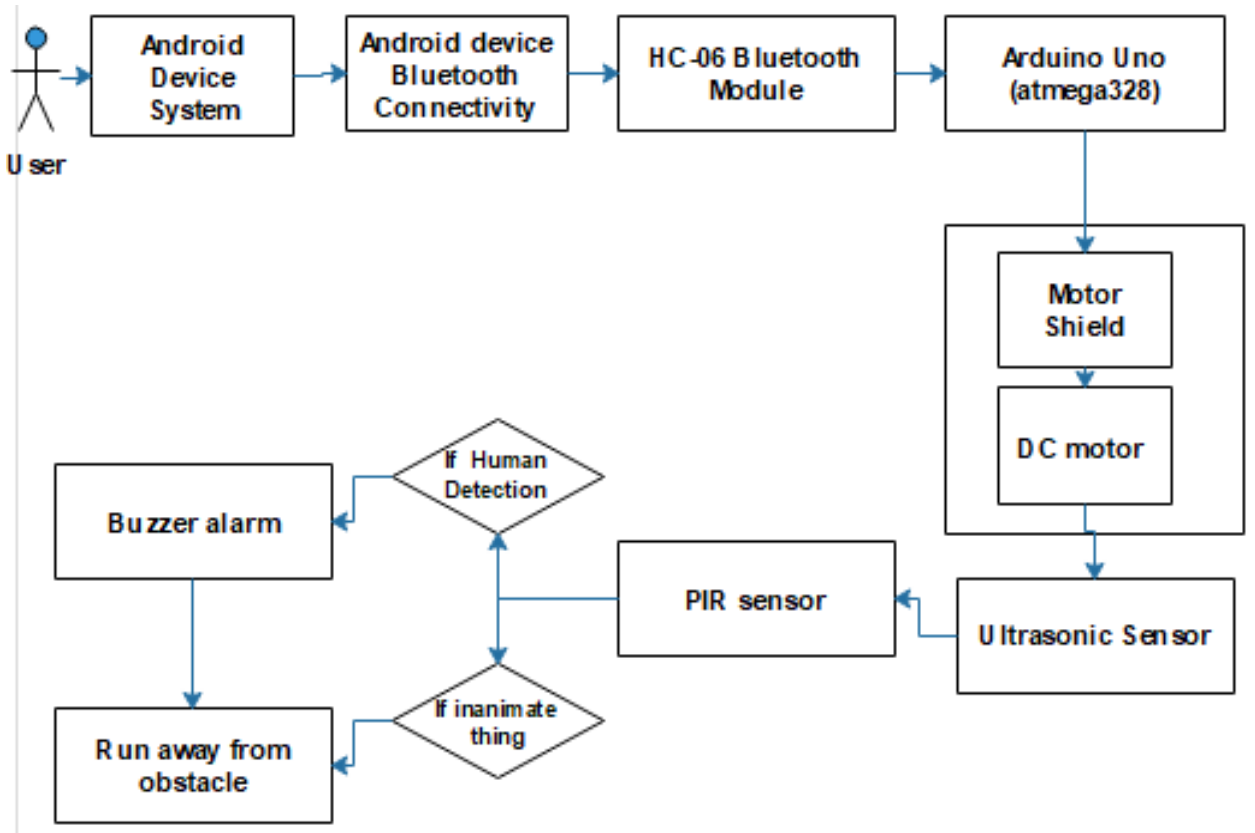

Figure 1. Structure of robotic car

\section{Technical Specifications of the Robotic Car}

\subsection{Arduino Uno}

Arduino Uno is the robot's brain. Arduino Uno uses the ATmega328 microprocessor. Arduino has 14 pins. The most popular Arduino card is the Arduino UNO model shown in Figure 2. It can be easily programmed with Arduino libraries [7]. Easily programmable is the biggest advantage of other microprocessors. Programming in the Arduino Uno is carried out specifically in the integrated development environment (IDE). The programming language is selected as Embedded C language. Using signals from sensors, it helps to design robots and systems that affect the environment. So actions happen such as sound and light specific to the output of the project.

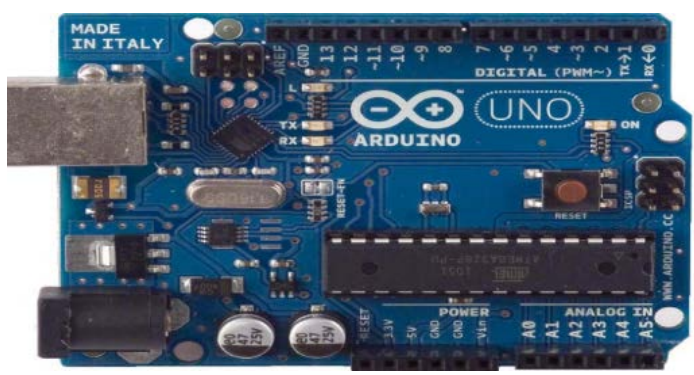

Figure 2. Arduino Uno

\subsection{Hc-06 Bluetooth Module}

The HC-06 Bluetooth module shown in Figure 3 provides communication between the devices at a short distance of approximately 10 to 20 meters. Communicates with Arduino via Serial Communication (USART). The Bluetooth module can only respond to incoming connection requests, so it can not send a connection request to another Bluetooth module. The Bluetooth module has a total of 4 pins. These are VCC, GND, Rx, and Tx.

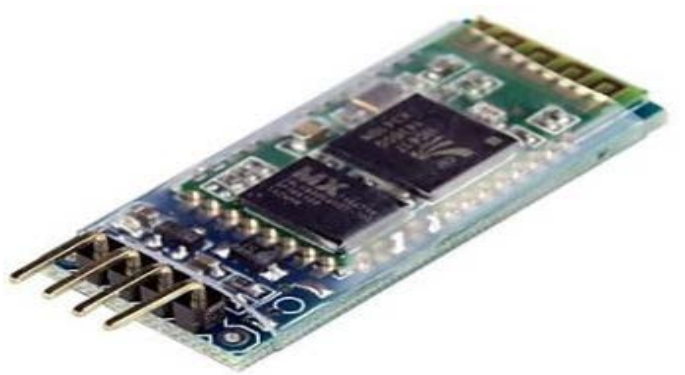

Figure 3. HC-06 Bluetooth Module

VCC and GND are supplied by Arduino for use in the Bluetooth module. Arduino's Tx pin must be plugged into the $\mathrm{Rx}$ part of the Bluetooth module so that commands from the Arduino can be detected by the Bluetooth module. Messages from Bluetooth need to be plugged into the Tx pin of the Arduino's Rx pin Bluetooth module in order to access Arduino [8]. A password is required to connect to the Android device via Bluetooth module.

\subsection{Buzzer}

The buzzer is a device which enables to produce various sound waves according to the voltage that is switched off. According to the market, it is cheap, easy to produce, and very lightweight. Therefore, the usage area is very large. The main purpose of the buzzer is to warn the authorities in 
relation to the identified situation. Thus, it takes various inputs and emits sound according to the inputs. First, the buzzer converts the dc voltage coming from the input port into the oscillation signal. The signal is amplified. When a piezo-discrete high voltage is applied, it causes mechanical expansion and contraction. This causes the inner metal plate to bend in the opposite direction. The metal plate is constantly twisted in the opposite direction (Figure 4), and the shrunk iceberg produces sound waves in the air.

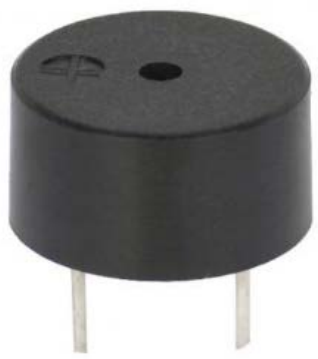

Figure 4. Buzzer

\subsection{Arduino Motor Shield}

Arduino Motor Shield is a motor driver card using L298 (Figure 5). This card is also designed to provide speed and direction control for DC Motor drive and to measure their current.

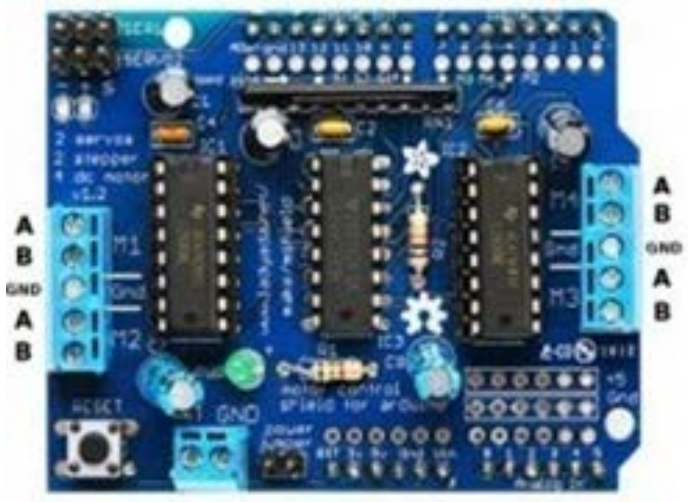

Figure 5. Motor Shield

\subsection{Motor}

Direct current (DC) motor converts electrical energy into mechanical energy using direct current [9]. DC motor is an electric machine that works with the principle of "A current carrying conductor is exposed to humid when it enters a magnetic field". The formula of the applied force is $\mathrm{F}=\mathrm{BIL}[10]$.

The main components of DC motors are consist of 6 pieces. These are coils, magnets, rotors, brush, stator and direct current source (Figure 6). In this study, the DC motor's purpose is to turn the wheels. The armature is placed in the magnetic field generated by the coils and rotated using direct current so that mechanical force is generated.

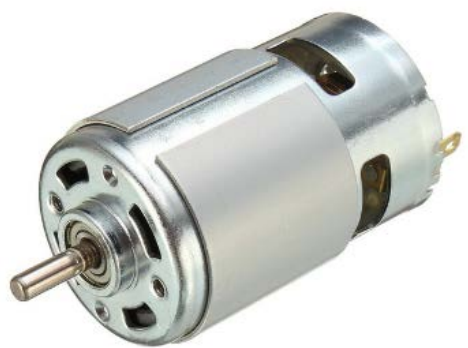

Figure 6. DC Motor

\subsection{PIR Sensor}

PIR (Passive Infrared sensor) sensor can detect a person's temperature. It is called passive infrared because it does not emit any heat or energy. This means that the PIR sensor uses infrared rays to detect human. The power consumption is low and the cost is also low.

The PIR sensor has two pin slots and each pin slot is IR sensitive (Figure 7) [11]. Thus, the visual distance of the sensor is determined. When the sensor sees nothing, the infrared emitted by all the objects in the room is at the same level and both slots are sensitive to IR level. Living things like humans enter the field of view of the sensor with the heat spread. This causes the temperature to change. If the object emits heat, the temperature of the sensor changes. The movement was perceived as a result of this change.

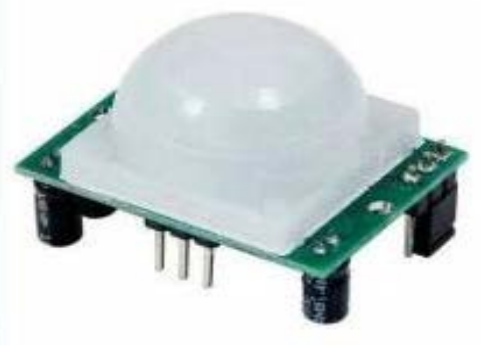

Figure 7. PIR Sensor

\subsection{Ultrasonic Sensor Hc-Sr04}

The HC-SR04 ultrasonic sensor uses Sonar (Sound Navigation and Variable) to calculate the distance to the object. Using sonar, the distance between robot and obstacle are measured. It makes the best measurement between $2-400 \mathrm{~cm}$.

Ultrasonic sound waves have frequencies between 20 $\mathrm{kHz}$ and $500 \mathrm{kHz}$. Ultrasonic sensors are sensors that emit ultrasonic sound waves and can determine the distance between them by calculating the time it takes for them to strike the obstacles (Figure 8). 


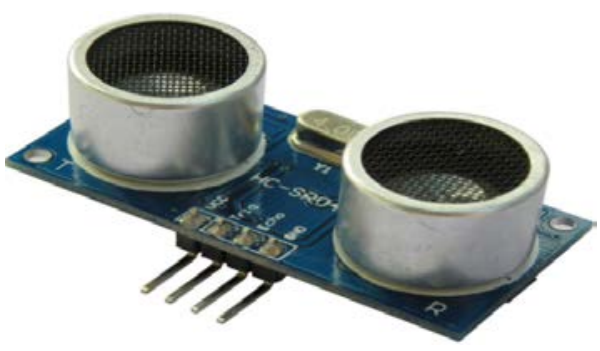

Figure 8. Ultrasonic Sensor HC-SR04

The sensing range of ultrasonic sensors can reach up to 30 meters under appropriate conditions. Ultrasonic sensors have two transducers. One of them is an ultrasonic speaker and the other is an ultrasonic microphone. With the electronic circuit, the time between the propagation of the sound wave from the ultrasonic loudspeaker and the detection of this sound wave by the obstacle and the reflection by the ultrasonic microphone is measured and the distance between the obstacle and the ultrasonic sensor is calculated as a result of dividing this time by the sound speed (Figure 9).

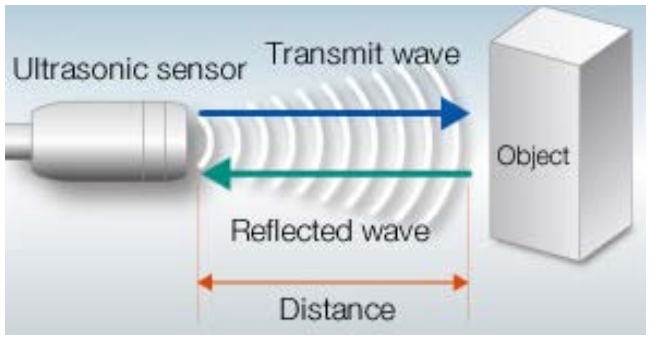

Figure 9. Ultrasonic Sensor working principle

\subsection{Arduino IDE}

The Arduino IDE is a software development platform that allows you to use the Arduino kits, write code, compile the code, and load the compiled code directly into the Arduino Uno (connected to the computer's USB port). In this platform C / C ++ languages are used. For C / C ++ languages, it consists of two general functions [9]:

- $\quad$ setup: this function starts working at the beginning of the program.

- loop: this function runs in a loop until the card power is off

\subsection{Android Platform}

The Android platform, which has a Linux-based operating system, is being developed by communities such as Google and the Open Handset Alliance. It is an operating system which is low cost and open source code. Originally used for tablets and smartphones. The Android operating system consists of 4 layers.

1- Linux Kernel

\section{2- $\quad$ Libraries \& Android Runtime \\ 3- Application Framework \\ 4- Applications}

The Kernel is the first layer of the Android operating system which is used for memory management, process management, and networking. The second layer is Libraries and Runtime which includes all libraries on the Android operating system are written in C and C ++. It is invoked via java interface. This layer works with the Dalvik virtual machine. This also serves as a kind of translator between the application and the operating system. The third layer is application framework which is used to determine the structure of an application for the Android operating system. The fourth and last layer is Applications which provides interaction between applications written in Java and users. Using the Bluetooth connection, the application is connected to the robot.

\section{Design and Implementation of the Robotic Car}

The robotic car is made up of an Arduino Uno, HC-06 Bluetooth module, Arduino motor shield, DC motor, Ultrasonic sensor HC-SR04, PIR sensor, buzzer, 9V battery (Figure 10).

In the implementation part, firstly the user should download the "Arduino Bluetooth Controller" from Google Play. After downloading the application, make sure that the Bluetooth connection is open. After entering the application, it must be connected with the Bluetooth module (HC-06) with "1234" password. After connecting to the application, the user should assign values to the desired keys. Once the assignment is done, the robots can now send input values.

The data sent from the Android application to the Arduino Uno with the Bluetooth module. Arduino Uno controls incoming signals and informs which signals should be transmitted to the motor driver. Thus, the robot moves in a certain order according to the inputs entered.

The user can control the basic movements of the robot, back and forth, right-left rotation, robot stop motion from their own intelligent device. The user can also take the robot in auto mode and allow the car to drive its own way. The robotic car can determine whether the obstacles across the vehicle are human. If there is an obstacle in existence, the red led on the robot lights up, the buzzer sounds an alarm, and the shortest distance that can be avoided is calculated and proceeded. If the obstacle facing the robot is an inanimate entity, it computes the shortest distance that it can avoid and proceeds in this direction. Furthermore, when the robot reaches the cliff, it perceives the abyss and stops itself (Figure 10). 


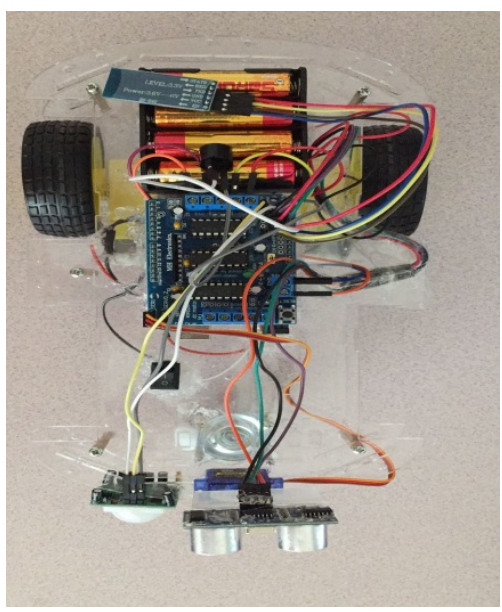

Figure 10. Robotic car top view

\subsection{User Control Mode and Automatic Mode}

To install the app, firstly, the user must have Google Play installed on his/her Android Device. If the user has Google Play installed, the user needs to download the application called Arduino Bluetooth Controller. To be able to connect to the application, Bluetooth must be turned on. After downloading the application, the user should connect the Bluetooth Module (HC-06) with using password "1234" Then, select the HC-06 and user will see some selection.

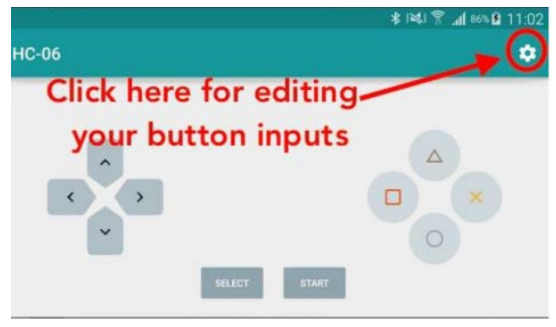

Figure 11. Buttons

Figure 11 shows how the user can change buttons on the Android application. As shown in the figure, when the user clicks on the symbol in the upper right corner, the user can assign the desired value to the buttons. When the user presses the Start button, the program starts running. The user can select the desired option by the select button. The options and values assigned in this study are as follows (Figure 12).

These five options provide the basic movements of the robotic vehicle. When the user selects the last option which is automatic mode button in the application, the robot will be controlled by itself. Thus, the robot will escape from the obstacles encountered and warn when it sees a living being.

In the first mode (manual), the user will be able to control the basic movement of the robot through the application. User-entered inputs will be forwarded to the robot via Bluetooth technology. The robot will receive inputs from the device, process them in the processor, Arduino Uno, and allow them to move according to the input order. The basic movements of the robot are forward, backward, right and left. In addition, the user can stop the robot at any time.

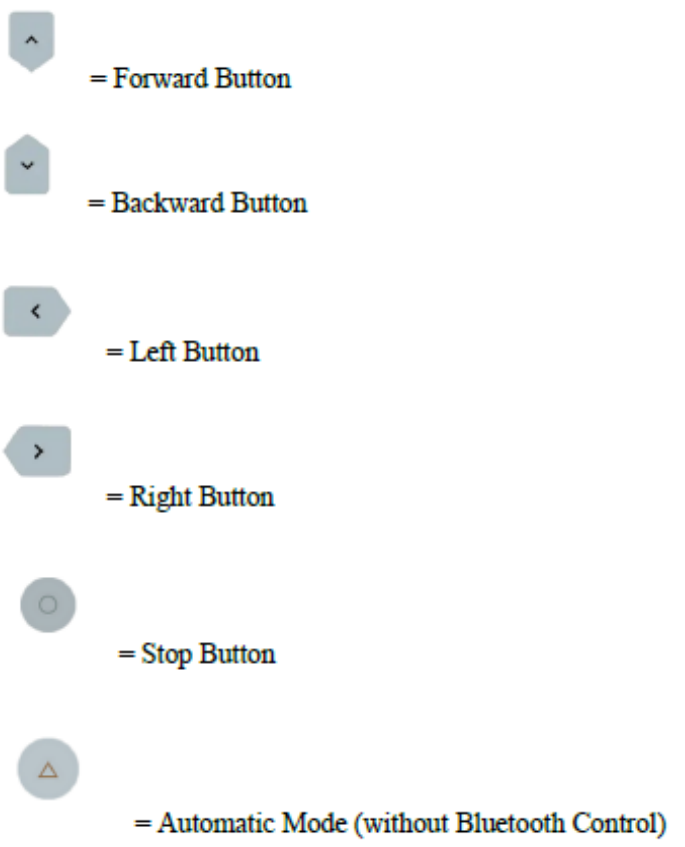

Figure 12. Assigned buttons

In the second mode (automatic), the user can take the robot in automatic mode by pressing the automatic mode key that the user sees in the interface of the application. In this mode, the robot will be able to control its basic movements. By means of the ultrasonic sensor, the obstacles are detected by Robot at $25 \mathrm{~cm}$ away. When the robot detects the obstacle, the robot stops itself and goes back $2 \mathrm{~cm}$. Then the PIR sensor will activate by the robot system and detect whether the obstacle is live or not. The robot computes the radiated heat from the obstacle. The object emits heat, and if it can move, the robot finds that the obstacle is alive. If the obstacle is a living entity the buzzer will active and an audible alarm sounds. Then the robot will escape from the obstacle and will continue on way. If the obstacle it perceives is an inanimate object, it computes the distance to the farthest point away from the obstacle and continues on its way by avoiding obstacles in that direction. This process continues until the user stops the robot and exits from the application. In addition, the user can configure the buttons in the application interface as desired. The user can give the desired values to the buttons.

In this study tracking path can be constituted by the user or by the automatic mode. According to selected option, 1robotic car can move with the instructions of the user with real time obstacle avoidance or 2- robotic car can move until it detects obstacle by using autonomous mode. 


\section{Conclusions}

There are many papers designed to address robotic vehicle with Arduino Uno, Raspberry pi and Android platform. However, real time obstacle detection and avoidance by using Arduino Uno and Android Platform of the remote and autonomous controlled robotic car has not been studied. The purpose of this article is to alert the user to potential terrorist attacks on the military field using the application on an Android device. By taking input from the Android application, the basic movements of the robot are provided. Communication between the robot and the application is made by the HC-06 Bluetooth module. The ultrasonic sensor HC-SR04 is used to prevent the robot from crashing. Thanks to this sensor, the robot manage to escapes the obstacles. In addition, when the robot comes near the cliff, it perceives it and saves itself from falling. With the help of the PIR sensor, the obstacle detected by the ultrasonic sensor is a person emitting heat is revealed. The audible alarm will start ringing with human detection. Novelty of this research is the use of an ultrasonic sensor to detect the distance of the robot's obstacle, avoid obstacles in front of inanimate objects, and determine human simultaneous detection. Thus, the commissioning of such a robot can improve its effective operation and control its tasks remotely and wirelessly, possibly resulting in an attack. This study can be further improved by using more talented materials. The Wi-Fi module can be used instead of the Bluetooth module. In this way, the Wi-Fi cannot be broken as fast as Bluetooth and can be used in a wider areas than Bluetooth.

\section{REFERENCES}

[1] S. S. Pujari, M. S. Patil, and S. S. Ingleshwar, "Remotely controlled autonomous robot using Android application", 2017 IEEE International Conference on I-SMAC (IoT in Social, Mobile, Analytics, and Cloud) (I-SMAC), 2017.

[2] M. R. Mishi, R. Bibi, and T. Ahsan, "Multiple motion control systems of the robotic car based on IoT to produce cloud service,” 2017 IEEE International Conference on Electrical, Computer and Communication Engineering (ECCE), 2017.

[3] D. Chakraborty, K. Sharma, R. K. Roy, H. Singh, and T. Bezboruah, "Android application based monitoring and controlling of movement of a remotely controlled robotic car mounted with various sensors via Bluetooth,” 2016 IEEE International Conference on Advances in Electrical, Electronic and Systems Engineering (ICAEES), 2016.

[4] S. J. Lee, J. Lim, G. Tewolde, and J. Kwon, “Autonomous tour guide robot by using ultrasonic range sensors and QR code recognition in an indoor environment," IEEE International Conference on Electro/Information Technology, 2014.

[5] E. Amareswar, G. S. S. K. Goud, K. R. Maheshwari, E.
Akhil, S. Aashraya, and T. Naveen, "Multipurpose military service robot,” 2017 IEEE International Conference of Electronics, Communication, and Aerospace Technology (ICECA), 2017.

[6] Premkumar, Keerthi, and K Gerard Joe Nigel. "Smart Phone-Based Robotic Arm Control Using Raspberry Pi, Android, and Wi-Fi.” 2015 IEEE International Conference on Innovations in Information, Embedded, and Communication Systems (ICIIECS), doi:10.1109/iciiecs. 2015.7192973, 2015.

[7] Varshney S., Gaur, B., Farooq O., Khan Y. U. "Brain Machine Interface for wrist movement using Robotic Arm”, IEEE 16th International Conference on Advanced Communication Technology, DOI: 10.1109/ICACT. 2014.6779014, 2014.

[8] Internet: Geleceği yazanlar, Bluetooth ile İletişim, https://gelecegiyazanlar.turkcell.com.tr/konu/arduino/egiti m/arduino-201/bluetooth-ile-iletisim, 20.06. 2018.

[9] Verma, S, Android App Controlled Bluetooth Robot, Internal Journal of Computer Applications, vol 152, no 9, p. $35-40,2014$

[10] Internet: Robotpark, DC Motor Nedir? http://www.robotpar k.com.tr/Dc-Motor-Nedir, 21.06.2018.

[11] Internet: Güvenlik Online, PIR Sensör, https://www.guvenli konline.com/makale/219/pir-sensor.html, 21.06.2018. 